\title{
A new stacking technique in ambient noise tomography*
}

\author{
Jing Liu Youcai Tang Kai Tao Chunquan Yu Jie Chen \\ Jieyuan Ning* Yongshun John Chen and Yongge Feng \\ Department of Geophysics, School of Earth and Space Sciences, Peking University, Beijing 100871, China
}

\begin{abstract}
We have developed a new stacking technique in ambient noise tomography to obtain high-quality dispersion curves of Rayleigh waves. This technique is used to stack the vertical components of the Estimated Green Functions (EGFs) obtained respectively from cross correlation of the ambient noise data recorded by a remote seismic station and one of the short distance seismic stations of a seismic array. It is based on a phase-matched filter and is implemented by a four-step iterative process: signal compression, stacking, signal extraction and signal decompression. The iterative process ends and gives the dispersion curve of Rayleigh wave when the predicted one and the processing result converge. We have tested the method using the vertical components of synthetic Rayleigh wave records. Results show that this new stacking method is stable and it can improve the quality of dispersion curves. In addition, we have applied this method to real data. We see that the results given by our new technique are obviously better than the ones employing the traditional method which is a three-step process: signal compression, signal extraction and signal decompression. In conclusion, the new method proposed in this paper can improve the signal to noise ratio of EGFs, and can therefore potentially improve the resolution of ambient noise tomography.
\end{abstract}

Key words: seismotectonics; continental dynamics; ambient noise tomography; array technique; stacking CLC number: P315.3 $\quad$ Document code: A

\section{Introduction}

Aki (1957) first proposed that the spatial correlation of ground motion was a zero degree Bessel function. Weaver and Lobkis (2001) showed that the wave forms obtained from the spatial correlation of two receivers' records behave as a Green's function originated from one station and received at the other station. The method has soon been applied to the seismotectonic studies (e.g., Campillo and Paul, 2003; Shapiro and Campillo, 2004).

Ambient noise tomography has several distinct advantages. First, it does not depend on the occurrence of earthquakes. Second, it can provide information in the shorter period range of $t<20 \mathrm{~s}$; whereas the energy of earthquake generated surface waves in this period range is much weaker at teleseismic stations because of attenuation. Therefore, ambient noise tomography has recently been used by many researchers to generate high resolution surface wave tomography maps of the Earth's

\footnotetext{
* Received 1 November 2010; accepted in revised form 15 November 2010; published 10 December 2010.

* Corresponding author. e-mail: njy@pku.edu.cn

(C) The Seismological Society of China and Springer-Verlag Berlin Heidelberg 2010
}

shallow structures (e.g., Shapiro et al., 2005; Yao et al., 2006; Yang et al., 2007; Zheng et al., 2008; Fang et al., 2009; Li et al., 2010).

Chinese mainland has complex geological structures and requires both high-quality data and new techniques to image it. North Chaina craton is an important unit in it and has attracted geoscientists' great attention. Recently, it was described as an activated or broken craton by geologists (e.g., Xu, 2001) and attracted great attention from geophysicists (e.g., Chen et al., 2006; Zheng et al., 2007; Chen, 2009; Zhao et al., 2009). Although earthquakes often hit this area, they are not evenly distributed. On the contrary, most part of the North China craton has been covered by evenly-spaced seismic arrays, which would be appropriate for executing ambient noise tomography. As more seismic stations are deployed in this region, ambient noise tomography will play a more important role in investigation of the shallow structure of Huabei craton.

Peking University has deployed four linear arrays which consist of more than 130 seismic stations in Huabei region since 2007. The longest array starts from Yinchuan city and ends at Jinan city (see Figure 1). Its 
length is about $1000 \mathrm{~km}$ and the average space between two neighbor stations is about $10 \mathrm{~km}$. The primary purpose of this $1000 \mathrm{~km}$-long seismic array was for receiver function studies (Tang et al., 2010a). However, recently it was also used to execute ambient noise tomography (Tang et al., 2010b).

As we all know that a reliable dispersion measurement at period $T$ requires interstation space of at least three wavelengths. Suppose phase speed is $4 \mathrm{~km} / \mathrm{s}$, around $5 \mathrm{~s}$ period seismic surface waves can be safely detected for a station distance being more than about 60 $\mathrm{km}$. If we want to employ greater period surface waves, the required station distance would be even longer. $\mathrm{Ob}$ viously we can not only use PKU array to do ambient noise tomography because a phase of PKU array only spans about $200 \mathrm{~km}$. However we can combine the observation data of the PKU array with those of Incorporated Research Institutions for Seismology (IRIS) for ambient noise tomography researches. In this way, the densely deployed seismic array can be used to enhance the signal to noise ratio (SNR) of the EGFs.

In this paper we propose a new stacking technique to use the data of densely deployed seismic stations and to obtain high quality EGFs of Rayleigh waves. We have tested the method using both synthesized Rayleigh waves and real seismic observations. Results show that the technique proposed in this paper can improve the signal to noise ratio of EGFs, and can therefore potentially improve the resolution of ambient noise tomography.

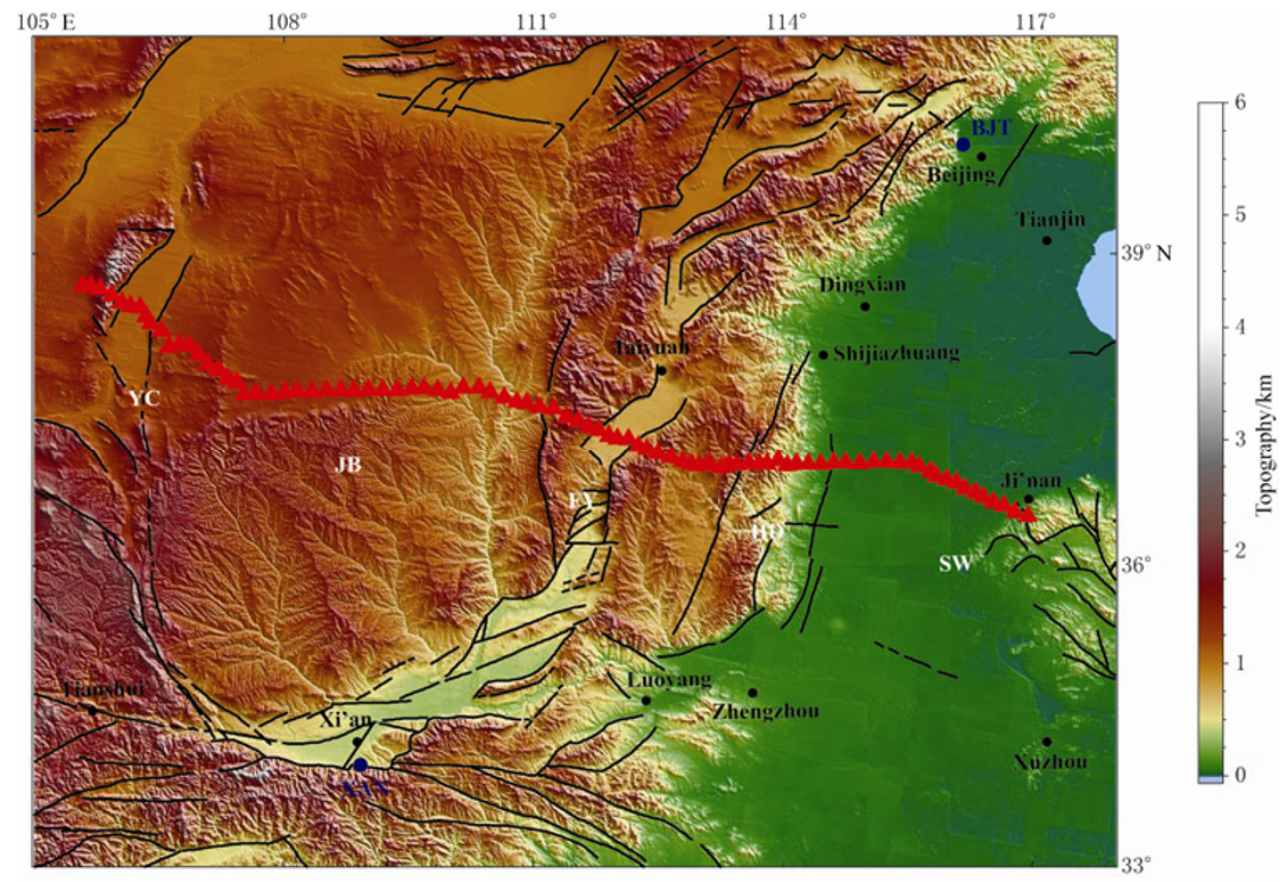

Figure 1 Locations of a PKU array and two IRIS stations. The red triangles are the temporary stations deployed by PKU in 2007-2010. The array consist of five parts: YC (Yinchuan profile), JB profile (Jingbian profile), FY (Fenyang profile), HD (Handan profile) and SW (west part of Shandong profile). Color image exhibits the topography of this area. The blue dots show the locations of two IRIS permanent stations: BJT and XAN.

\section{Stacking: An efficint array technique to improve SNR}

Mostly because we did not have enough seismographs, we could only deploy limited length of profile in a phase of observation when we built an array with small interstation interval. Thus we cannot only use the array data to do ambient noise tomography because they do not satisfy interstation distance requirement men- tioned above. However we can correlate the observations of every station in an array with those of a remote station.

Furthermore, when two neighbor stations are near with each other, the lithospheric structures beneath them are mostly very similar, so do the two EGFs of Rayleigh waves respectively given by cross-correlation between the data recorded by the first or the second station with the data recorded by a remote station. Mostly the differ- 
ences in frequency dispersion curves of the two EGFs of Rayleigh waves are introduced by interference like local events. Accordingly we propose stacking them for suppressing interference and extracting dispersion curves of Rayleigh waves with high SNR. In this way, we might not only enlarge the ambient noise database, but also contribute higher quality data for ambient noise tomography.

However we cannot just simply stack the EGFs of Rayleigh waves obtained by two-station cross-correlation unless the two neighbor stations are on a circle with the remote station as their center owing to dispersion characteristics of the Rayleigh waves. For overcoming this difficulty, we need execute stacking after applying anti-dispersion. The compressed signals after executing anti-dispersion are supposed to contain no information for ray propagation and would be the EGFs of Rayleigh waves without dispersion and are appropriate for executing stacking.

In the traditional three-step group velocity measurement procedure including signal compression, signal extraction and signal decompression (hereafter we call it three-S method), which was proposed by Herrin and Goforth (1977), developed by Levshin and Ritzwoller (2001) and widely employed in ambient noise tomography (e.g., Shapiro et al., 2005; Yang et al., 2007; Zheng et al., 2008), actually includes anti-dispersion processing in it. So, we only need change the three-step velocity measurement procedure into four-step one, which includes signal compression, stacking, signal extraction and signal decompression (hereafter we name it as four-S method).

As mentioned above, when the distance between $A$ and the center of two neighbor stations $B$ and $C$ is much bigger than the distance between $B$ and $C$, the two compressed EGFs of Rayleigh wave could be similar because the two wave paths are very close to each other. Certainly if the structures along the two wave paths are significantly different, the two compressed EGFs of Rayleigh wave would also be much different. In this condition, stacking could not help to create a more delta-like curve. However it can be used to detect the abrupt changes between the wave paths from $A$ to $B$ and from $A$ to $C$. All in all, the most important thing is that the distance between $B$ and $C$ must be small. When we have a linear array and one or more remote stations which are nearly perpendicular with the trend of the array, we can use above method both to obtain more accurate dispersion curves and to find the places with ab- rupt velocity changes.

It needs mention that above procedure is an iteration process. We do iteration until the root-mean-square (RMS) error between the current dispersion curve and the prior one reaches or less than $5 \%$. This procedure assures that we get consistent and stable results.

\section{Testing: Practices on simulated data}

For testing our method, we firstly obtain the simulated data (or synthetic seismic data). Full-wavefield seismograms were calculated by qseis 06 program using the reflectivity method (Wang, 1999). The algorithm solved the numerical difficulty in the original Thomson-Haskell propagation by adopting a numerical procedure that makes the fundamental displacement vector in situ orthonormal. The Earth flattening approximation (Mueller, 1977) is used to account for the Earth's curvature. The method is efficient and stable for a wide range of applications from calculation of static deformation to generating high-frequency seismograms (Wang, 1999).

The three imaginary stations with $0.1^{\circ}$ interval have the same underground velocity structure, while the source is $2^{\circ}$ away from the first station. We place the source at the Earth's surface with vertical motion as its only non-zero component. The source time function is Heaviside function. In order to get pure surface wave, we chose the slowness taper to filter the body waves, but there could still be some body wave energy remained in the outputs.

We do the same disposals on the simulated data as those that people normally executed on real ambient noise data, but here we execute stacking mentioned above. After applying anti-dispersion on the cross correlation results between three neighbor stations and a remote station respectively, we stack the three compressed signals. Then we execute signal extraction (for obtaining cleaned signal) and signal decompression. At last we obtain the dispersion curve of the route from the remote station to the center station of the three neighbor stations after iteration (refer to Figure 2).

Computation results show that the dispersion curve which only used ordinary frequency time analysis (FTAN) from the theoretical synthetic surface waves of the center station is much similar to the ones executing phase matched filtering for cases with and without employing stacking when the width of temporal window of signal extraction is narrow (see Figure 2 ); but when we augment the temporal window of signal extraction or do not use it, comparing with the one 

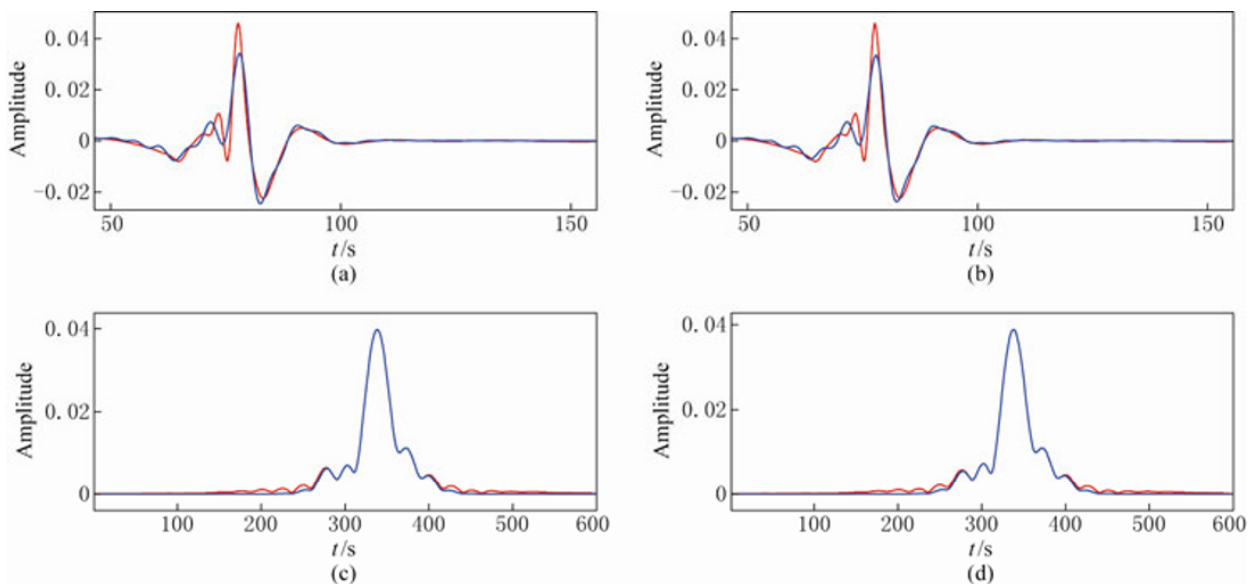

(c)

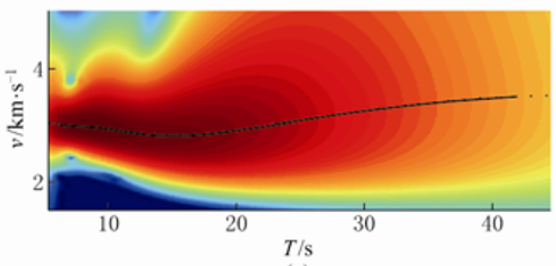

(e)

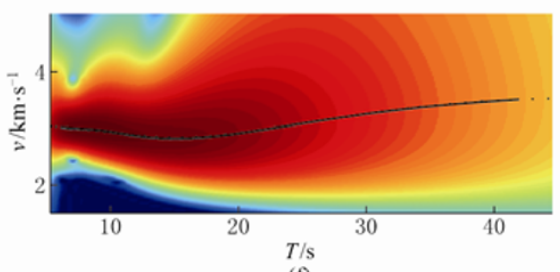

(f)

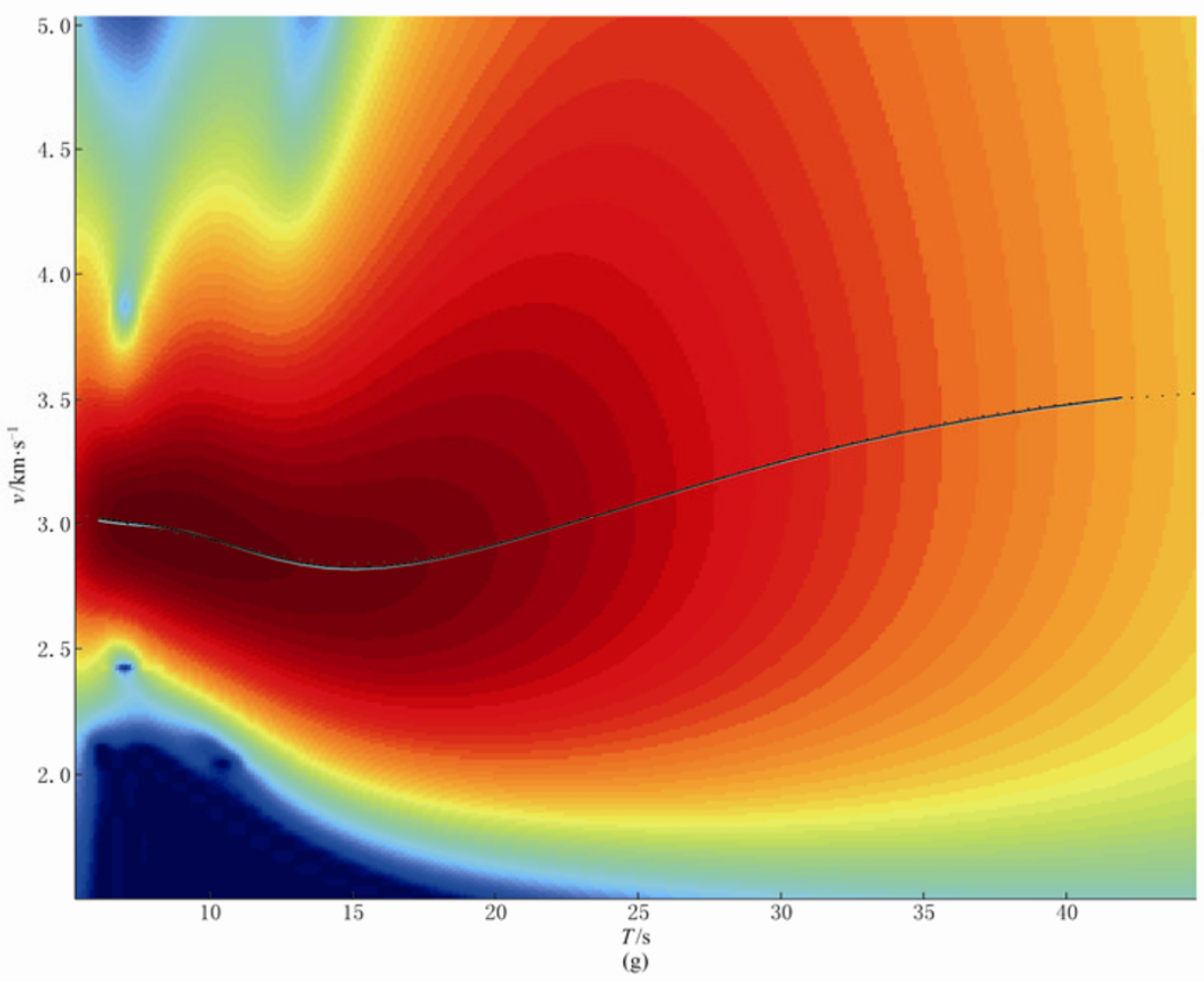

Figure 2 Graphical representation of FTAN of pure synthetic data with small temporal window of signal extraction. (a) Raw (red) and cleaned (blue) waveforms of synthetic Rayleigh wave at the central station without employing stacking. (b) Raw (red) and cleaned (blue) waveforms for synthetic Rayleigh wave at the central station using the method proposed in this paper. (c) Compressed signal (red) and its corresponding cleaned signal (blue). (d) Compressed signal after executing stacking (red) and its corresponding cleaned signal (blue). (e) Cleaned FTAN diagram, measured group speed curve using three-step method (solid line), the result given by ordinary FTAN on the raw data is also shown in this figure (dashed line), color image shows normalized energy of the signal. (f) Cleaned FTAN diagram, measured group speed curve using four-step method (black solid line), the one employing three-step method (green solid line) and the one given by ordinary FTAN on raw data (dashed line) are also shown in this figure. (g) The amplified Figure $2 \mathrm{f}$. 
without employing stacking, the dispersion curve implementing stacking is closer to the dispersion curve

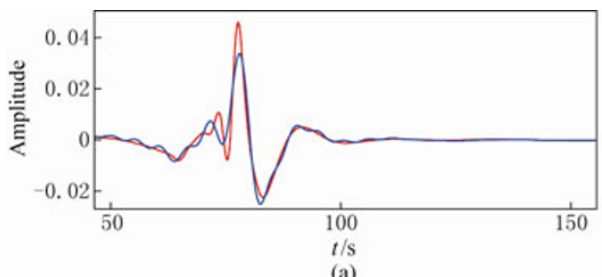

(a)
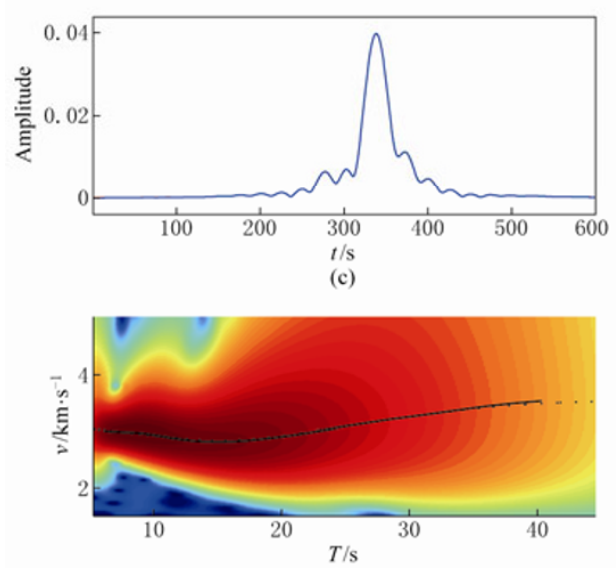

which only used FTAN from the synthetic surface waves (see Figure 3).

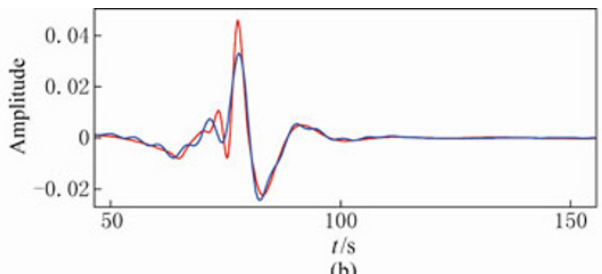

(b)

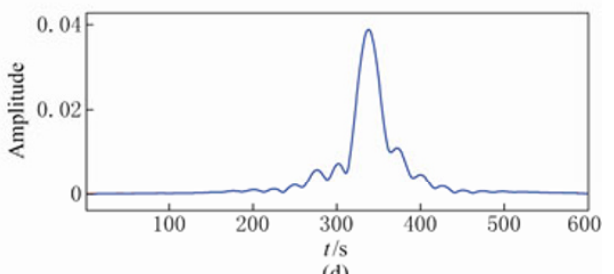

(d)

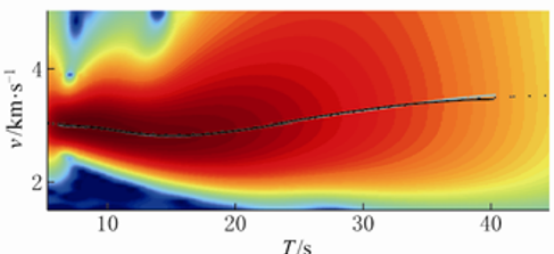

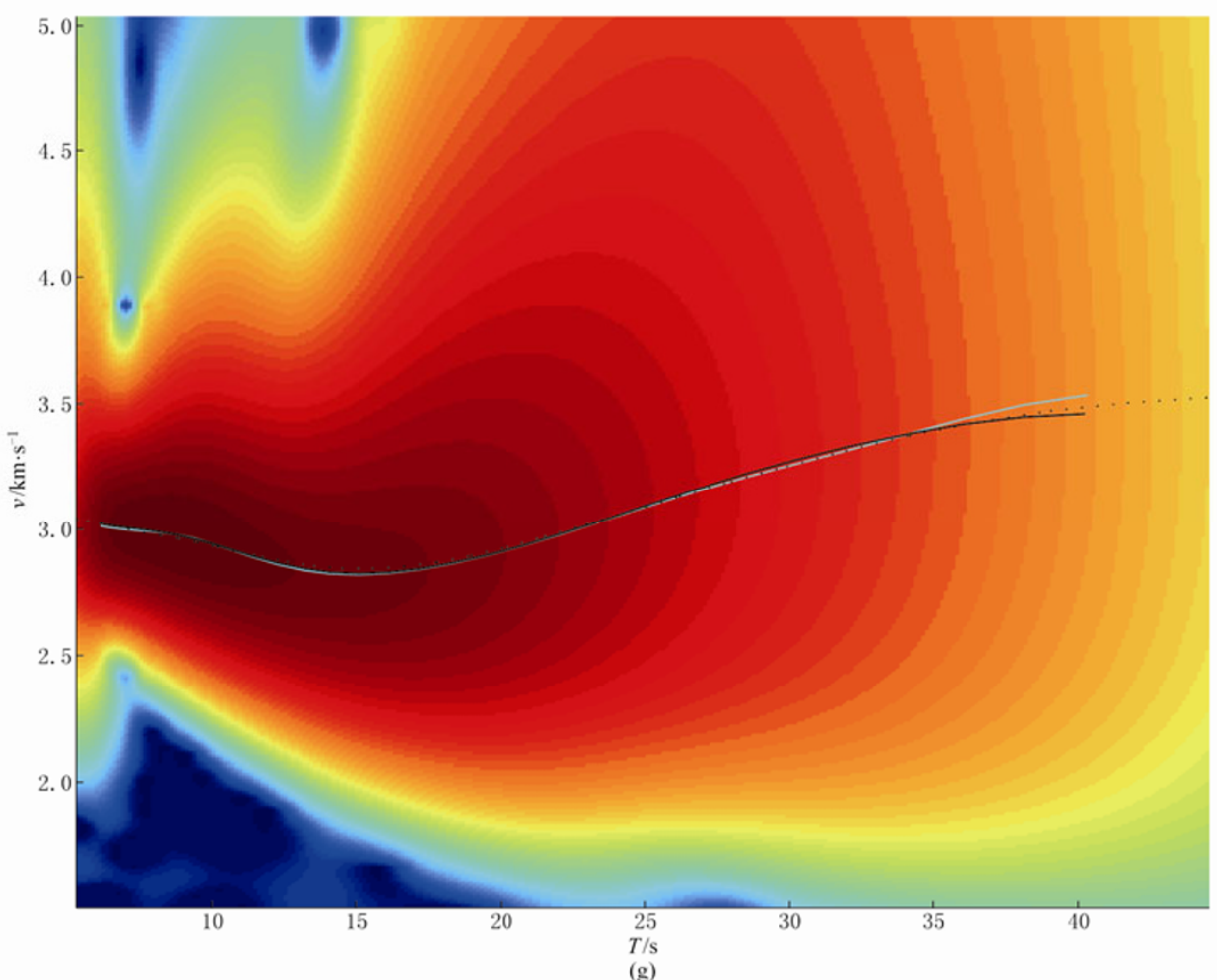

Figure 3 Graphical representation of FTAN of pure synthetic data for greater temporal window of signal extraction. The pictures have the same meaning with Figure 2, but the fixed width of temporal window of signal extraction employed is much wider than that in Figure 2.

Then we use Matlab function rand to add white noise into the theoretical records and write them into a
SAC format file. In order to let the records behave more like real data, we have filtered the records use 3.3-50 s 
filter. When the width of temporal window of signal extraction is wider, the center station's dispersion curve executing stacking is much closer to that got from

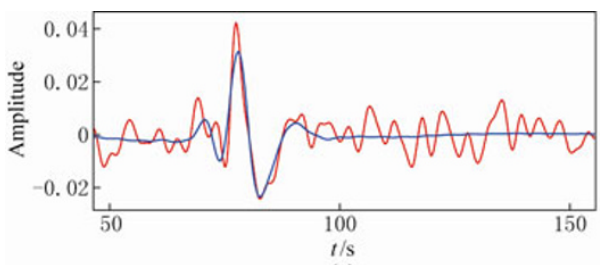

(a)
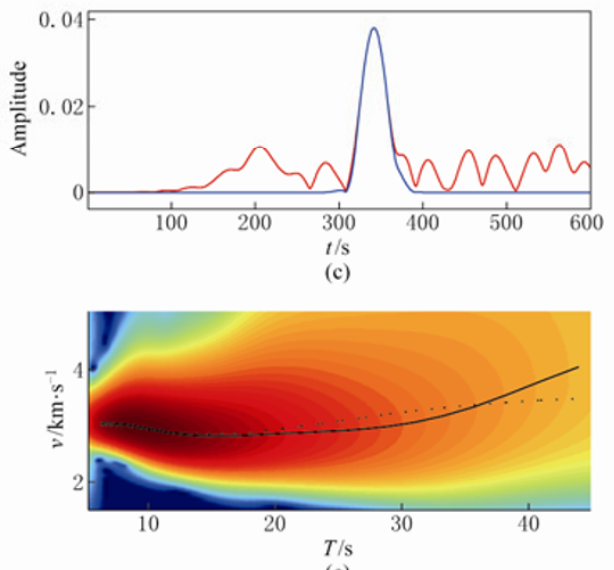

(e)
FTAN using the synthetic surface waves than the one without stacking (refer to the cleaned FTAN diagram Figure 4).

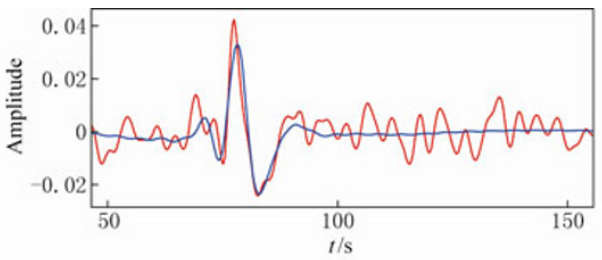

(b)

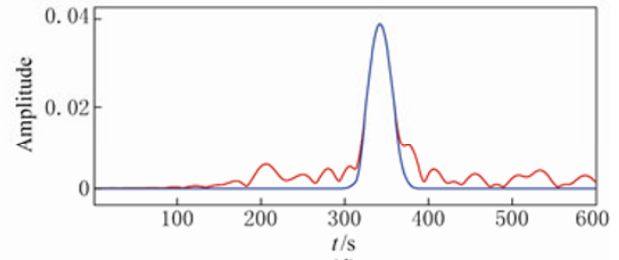

(d)

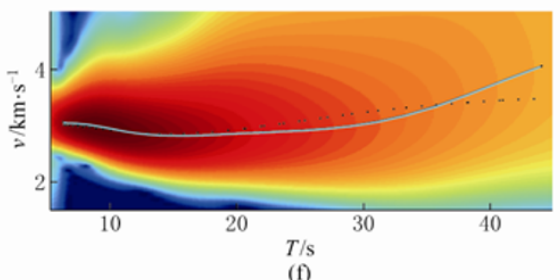

(f)

Figure 4 Graphical representation of FTAN of synthetic data with noise added for wide temporal window of signal extraction. (a) Raw (red) wave form and cleaned waveform using three-step method (blue) for the theoretical synthetic surface wave with noise added at the central station. (b) Raw wave form (red) and cleaned waveform (blue) for the theoretical synthetic surface wave with noise added at the central station using four-step method. (c) Compressed signal (red) and its corresponding cleaned signal (blue) given by three-step method. (d) Compressed signal (red) and its corresponding cleaned signal (blue) given by four-step method. (e) Cleaned FTAN diagram, measured group speed curve using three-step method (solid line) and the one got only from the ordinary FTAN using the synthetic surface wave (dashed line). (f) Cleaned FTAN diagram, measured group speed curve using four-step method (black solid line), threee-step method (green solid line) and the one got only by the ordinary FTAN on the synthetic surface wave (dashed line).

However when the width of temporal window of signal extraction is narrow enough, the dispersion curves got from both with and without spatial stacking are much similar, but both are deviate from the dispersion curves only used FTAN from the synthetic surface waves (refer to the cleaned FTAN diagram in Figure 5). We can see that when we execute stacking, the dependence of the results on fixed temporal window is reduced. As we all know that, the width of the window should be broad enough to encompass the broadening of the compressed signal caused by the difference between the predicted and real dispersion curves, but it should also be narrow enough to filter out unwanted signals. That is to say, the wider we choose the fixed temporal window, the more signal we get, but with more noise. Of course if we want to get much better result, we still need to choose the appropriate fixed temporal window of signal extraction, which is determined by the structure that we investigate. In general, the more complex the structure, the wider the fixed temporal window we will choose.

\section{Applications in a PKU Seismic Array}

Peking University has deployed four profiles which are consisted of more than 130 seismic stations in Huabei area since 2007. The longest array starts from Yinchuan and ends with Ji'nan, which has five parts: YC, JB, FY, HD and SW. Limited by the number of seismographs, these stations were deployed in different time periods. Mostly they had one-year observation, however until now SW only had seven months records. We choose some IRIS seismic stations in China (e.g., BJT, XAN, ENH, MDJ, ULN, HIA, KMI, QIZ, CHTO), as 

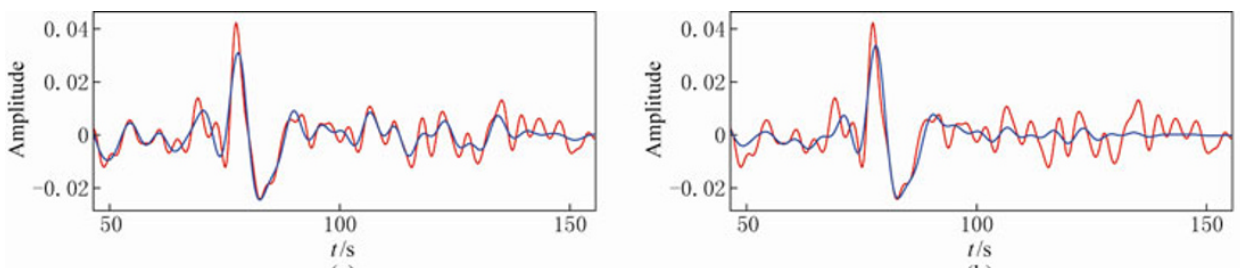

(a)
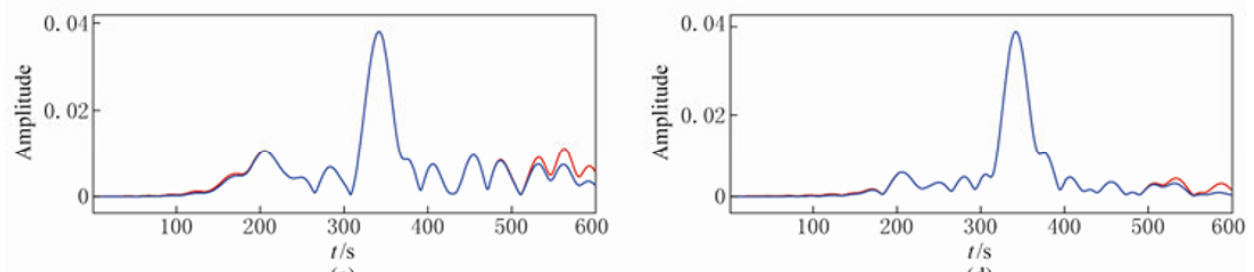

(d)
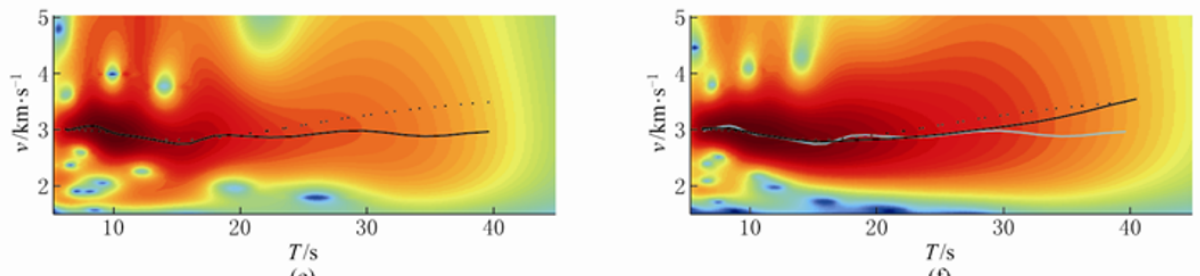

(e)

(f)

Figure 5 Graphical representation of FTAN of synthetic data with noise added for narrow temporal window of signal extraction. (a) Raw (red) and cleaned (blue) waveforms for the theoretical synthetic surface wave with noise added at the central station using three-step method. (b) Raw (red) and cleaned (blue) waveforms for the theoretical synthetic surface wave with noise added at the central station using four-step method. (c) Compressed signal (red) and its corresponding cleaned signal (blue) just using three-step method. (d) Compressed signal (red) and its corresponding cleaned signal (blue) using four-step method. (e) Cleaned FTAN diagram, measured group speed curve just using three-step method (solid line) and the one got only from the ordinary FTAN using the synthetic surface wave (dashed line). (f) Cleaned FTAN diagram, measured group speed curve using four-step method (black solid line), three-step method (green solid line) and the one got only from the ordinary FTAN using the synthetic surface wave (dashed line).

remote stations, which constitute station pairs separately with the stations of the PKU array. The two IRIS stations BJT and XAN which are used to demonstrate the robustness of our method are shown in Figure 1.

As a normal procedure in ambient noise analyses, we execute cross-correlation, but only for station pairs which are composed of a remote station and a station of the PKU array. The two stations of a station pair should have the same observation time period. However, owing to the objectivity of the results of cross-correlation, there would be no need to consider their time consistence after we have obtained the cross-correlation results.

Here we select station pairs which are composed of one fixed IRIS station (remote station) and one of the three neighboring PKU stations to do stacking. According to above discussion, as long as these three neighboring stations are very close to each other, we can execute stacking. We take the stacking result as the input of signal extraction and then do redispersion. As last we get cleaned a FTAN diagram of the middle station by our new four-S method.

As mentioned above, we got the last dispersion curve through iteration when the RMS error between the current one and the prior one reaches or less than 5\%. Considering the PKU array is a linear array roughly with east-west trending, we select the three-station group sequentially from west to east. Except the west-end station and the east-end station, all other stations have one opportunity to be the middle station.

While underground velocity structure varies smoothly, stacking should improve the signal to noise ratio. If there is abrupt velocity change in the path between the remote station and the three PKU neighboring stations, stacking effect would be bad and it can be used to detect the tectonic boundary.

In Figures 6 and 7 we show the results obtained from a remote station BJT and the two stations of PKU array. As shown in the Figure 6, for the data observed in 
12 months the stacked correlation result of three neighboring stations is obviously better than the one without stacking. The qualities of the compressed signal and the cleaned FTAN diagram given by the four-S method are obviously better than those given by three-S method.
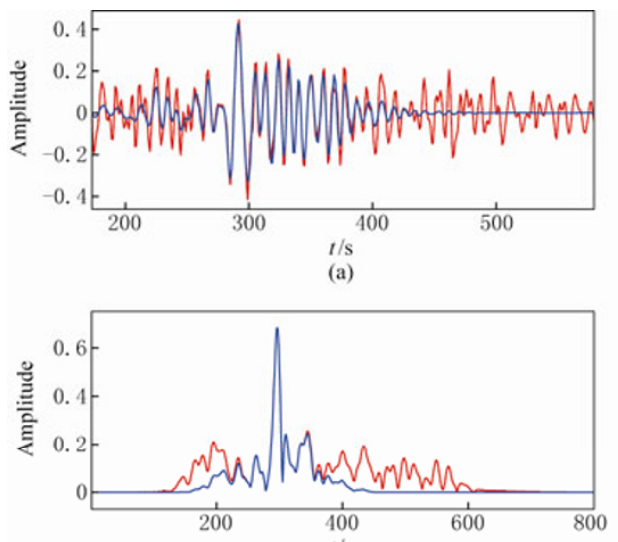

(c)

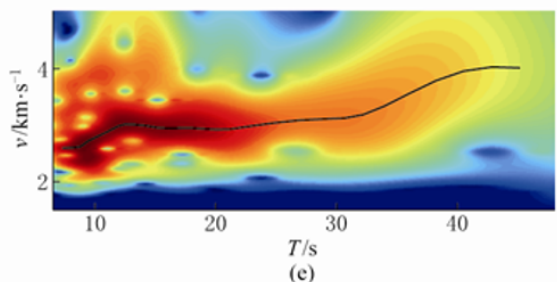

Furthermore, the stacked result of six-months data recorded by three neighboring stations is also obviously better than the one without stacking and fits the result of the center station's non-stacking result given by one year records very well (refer to Figure 7).
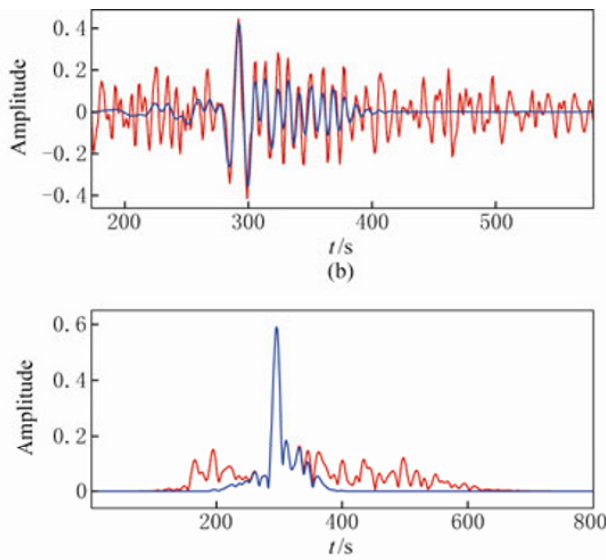

(d)

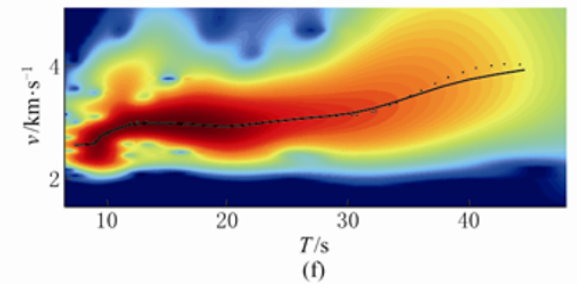

Figure 6 Graphical representation of FTAN of real data observed in 12 months. (a) Raw waveform (red) obtained from 12-month stacked cross-correlation between stations BJT and YC06 and the cleaned waveform (blue) obtained by three-step method. (b) Raw waveform (red) obtaind from the 12-month stacked cross-correlation between stations BJT and YC06 and cleaned waveform (blue) using four-step method. (c) Compressed signal (red) and its corresponding cleaned signal (blue) using three-step method. (d) Compressed signal (red) and its corresponding cleaned signal (blue) using four-step method. (e) Cleaned FTAN diagram, measured group speed curve using three-step method. (f) Cleaned FTAN diagram, measured group speed curve using four-step method (solid line) and three-step method (dashed line).

\section{Discussion and conclusions}

Cross correlation of ambient noise records is now routinely used to get dispersion curves, and then do seismic tomography; however little attention has been paid to array techniques in ambient noise tomography. In this study we employ stacking technique for obtaining better dispersion curves and develop a four-S method. We test the method using the synthetic seismic surface wave records with and without noise added and find that our method is stable and has obviously improved the quality of dispersion curves. Then we use the data of PKU array with a station spacing of about $10 \mathrm{~km}$, and the IRIS stations far away from PKU array, to test the method. Results showed that our method is a robust technique in ambient noise tomography.
Generally speaking, the farther the distance between two stations, the more the unrelated signal will include, and the worse the correlation will be. Our method is one of the solutions to overcome this difficulty and can improve the signal to noise ratio of the EGFs and therefore, can potentially enlarge the dataset and improve the data quality, which can be used to reveal the complex structure of the Huabei craton or Chinese mainland .

Geophysicists have recently deployed many seismic arrays in China. For example, Peking University has deployed four profiles which are consisted of more than 130 seismic stations in Huabei area since 2007. Our method presented here should help us fully employ these seismic arrays in revealing the complex structure of the Chinese mainland. 

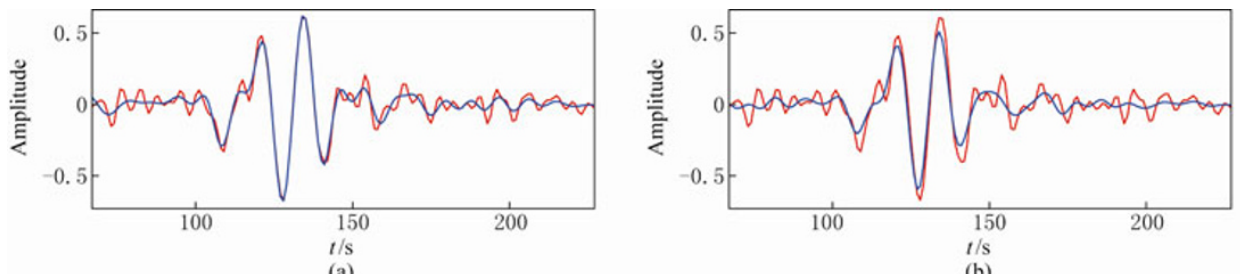

(a)
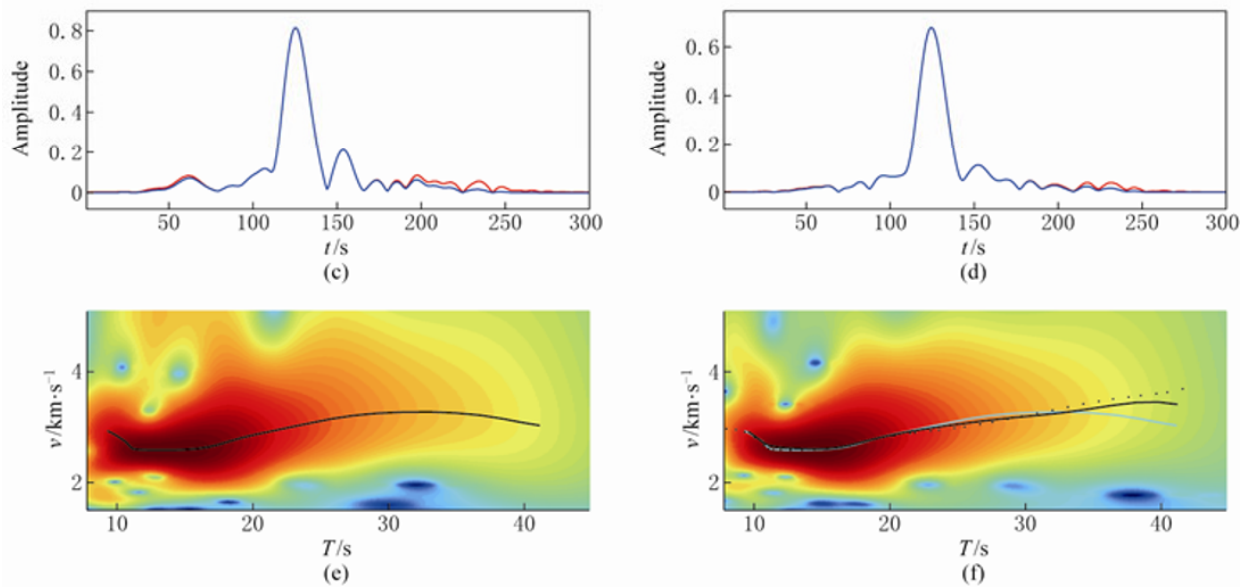

Figure 7 Graphical representation of FTAN of real data observed in six months. (a) Raw waveform (red) obtained by six-month stacked cross-correlation between stations BJT and HD20 and the cleaned one (blue) obtained from three-step method. (b) Raw waveform (red) obtained from six-month stacked cross-correlation between stations BJT and HD20 and the cleaned (blue) waveform using four-step method. (c) Compressed signal (red) and its corresponding cleaned signal (blue) using three-step method. (d) Compressed signal (red) and its corresponding cleaned signal (blue) using four-step method. (e) Cleaned FTAN diagram, measured group speed curve using three-step method. (f) Cleaned FTAN diagram, measured group speed curves using four-step method in six-month (black solid line), three-step method in six-month (green solid line) and three-step method in 12-month (black dashed line).

Acknowledgements The authors thank Yingjie Yang, Sihua Zheng and Zhen Xu for their technical assistance with the ANA program, Xiaodong Song, Yongen Cai, Shiyong Zhou for helpful discussions, PKU fieldwork group including Xiaofeng Liang, Han Yue, Mingming Jiang, Xiaoting Lou, Chunquan Yu, Shuguang Wang et al. for data collection. We also thank anonymous reviewers for their helpful comments and suggestions on the manuscript. This work was supported by the National Natural Science Foundation of China with grant No. 90814002 and 40821062 as well as Institute of Crustal Dynamics with grant No. ZDJ2008-18.

\section{References}

Aki K (1957). Space and time spectra of stationary stochastic waves, with special reference to microtremors. Bull Earthquake Res Inst Univ Tokyo 35: 415-457.

Campillo M and Paul A (2003). Long-range correlations in the diffuse seismic coda. Science 299: 547-549.

Chen L, Zheng T Y and Xu W W (2006). A thinned lithospheric image of the Tanlu Fault Zone, eastern China: Constructed from wave equation based receiver function migration. $J$ Geophy Res 111: B09312, doi: 10.1029/2005JB003974

Chen L (2009). Lithospheric structure variations between the eastern and central North China Craton from S- and P-receiver function migration. Phys Earth Planet Inter 173: 216-227.

Fang L H, Wu J P and Lu Z Y (2009). Rayleigh wave group velocity tomography from ambient seismic noise in North China. Chinese J Geophys 52(3): 663-671 (in Chinese with English abstract).

Herrin E E and Goforth T T (1977). Phase-matched filters: Application to the study of Rayleigh waves. Bull Seismol Soc Am 88: $744-757$.

Levshin A L and Ritzwoller M H (2001). Automated detection, extraction, and measurement of regional surface waves. Pure Appl Geophys 158(8): 1 531-1 545.

Li Y, Yao H J, Liu Q Y, Chen J H, van der Hilst R D, Li S C, Huang H, Guo B, Wang J and Qi S H (2010). Phase velocity array tomography of Rayleigh waves in western Sichuan from ambient seismic noise. Chinese J Geophys 53(4): 842-852, doi: 10.3969/j.issn.0001-5733.2010.04.009 (in Chinese with English abstract).

Mueller G (1977). Earth-flattening approximation for body waves from geometric ray theory: Improvements, corrections and 
range of applicability. $J$ Geophys 42: 429-436.

Shapiro N M and Campillo M (2004). Emergence of broadband Rayleigh waves from correlations of the ambient seismic noise. Geophys Res Lett 31, doi: 10. 1029/2004GL019491.

Shapiro N M, Campillo M, Stehly L, and Ritzwoller M H (2005). High-resolution surface-wave tomography from ambient seismic noise. Science 307: 1615-1 618.

Tang Y C, Feng Y G, Chen Y S, Zhou S Y, Ning J Y, Wei S Q, Li P, Yu C Q, Fan W Y and Wang H Y (2010a). Receiver function analysis at Shanxi Rift. Chinese J Geophys 53(9): 2 102-2 109, doi: 10.3969/j.issn.0001-5733.2010.09.010 (in Chinese with English abstract).

Tang Y C, Chen Y S, Yang Y J, Ding Z F, Liu R F, Feng Y G, Li P, Yu C Q, Wei S Q, Fan W Y, Wang H Y, Zhou S Y and Ning J Y (2010b). Ambient noise tomography in North China craton. Submitted to Chinese J Geophys (in Chinese with English abstract)

Wang R (1999). A simple orthonormalization method for the stable and efficient computation of Green's functions. Bull Seismol Soc Am 89: 733-741.

Weaver R L and Lobkis O I (2001). On the emergence of the Green's function in the correlations of a diffuse field. $J$
Acoust Soc Amer 110: 3011-3 017.

$\mathrm{Xu}$ Y G (2001). Thermo-tectonic destruction of the archaean lithospheric keel beneath the sino-korean craton in china: evidence, timing and mechanism. Phys Chem Earth (A) 26(9-10): 747-757.

Yang Y J, Ritzwoller M H, Levshin A L and Shapiro N M (2007). Ambient noise Rayleigh wave tomography across Europe. Geophys J Int 168: 259-274.

Yao H, van der Hilst R D and de Hoop M V (2006). Surface-wave array tomography in SE Tibet from ambient seismic noise and two-station analysis-I. Phase velocity maps. Geophys J Int 166: 732-744.

Zhao L, Allen R M, Zheng T and Hung S H (2009). Reactivation of an Archean craton: Constraints from P- and S-wave tomography in North China. Geophys Res Lett 36: L17306.

Zheng S H, Sun X L, Song X D, Yang Y J and Ritzwoller M H (2008). Surface wave tomography of China from ambient seismic noise correlation. Geochem Geophys Geosyst 9: doi: 10.1029/2008GC001981.

Zheng T Y, Chen L, Zhao L and Zhu R X (2007). Crustal structure across the Yanshan belt at the northern margin of the North China Craton. Phys Earth Planet Inter 161: 36-49. 